\section{Comparative genomics Grasping human transcriptome evolution: what does it all mean?}

MC Oldham and DH Geschwind

Heredity (2006) 96, 339-340. doi:10.1038/sj.hdy.6800807; published online 22 March 2006

$\mathrm{T}$ he recent publication of the chimpanzee genome sequence has confirmed its $96 \%$ identity to our own (Cheng et al, 2005; Consortium, 2005). The race is now on to determine which of these differences are responsible for our uniquely human features. In work published recently in Science, Khaitovich et al (2005) try to bring us closer to this goal by exploring broad evolutionary patterns for thousands of genes by comparing them between humans and chimpanzees both in terms of expression level and protein-coding sequence for the first time. The authors conclude that genes evolve in both of these dimensions in parallel as a function of their tissue environment, with some tissues imposing greater evolutionary constraint than others, and brain the most.

The authors used microarrays to measure expression levels for $\sim 21000$ genes in heart, kidney, liver, testis, and brain (prefrontal cortex) from six humans and five chimpanzees. For each tissue, they assessed the diversity of gene expression within species and the divergence of gene expression between species. Surprisingly, the authors found that human and chimpanzee gene expression patterns were much more similar in the brain than they were in other tissues. In addition, the ratio of gene expression divergence between species to diversity within species - a putative measure of positive selection (Khaitovich et al, 2004) - was significantly higher in testis compared to other tissues. The authors demonstrate that these effects do not derive solely from tissue-specific genes, but are also evident to a lesser extent in genes that are expressed in multiple tissues (albeit at different levels).

Khaitovich and colleagues also estimated the protein-coding sequence divergence between humans and chimpanzees for genes expressed in at least one tissue by calculating a measure of protein evolution, the $\mathrm{Ka} / \mathrm{Ki}$ ratio $(\mathrm{Ka}$ is the nonsynonymous substitutions/ nonsynonymous site; $\mathrm{Ki}$ is the synony- mous substitutions/synonymous site). Low $\mathrm{Ka} / \mathrm{Ki}$ values suggest strong constraint on protein evolution, whereas high $\mathrm{Ka} / \mathrm{Ki}$ values suggest either reduced constraint or positive selection (Fay and $\mathrm{Wu}, 2003$ ). The authors observed that the $\mathrm{Ka} / \mathrm{Ki}$ ratio was significantly lower for brain-specific genes than for all other tissue-specific genes. In addition, ubiquitously expressed genes showed lower $\mathrm{Ka} / \mathrm{Ki}$ ratios than genes expressed in a single tissue. Thus, in terms of both gene expression and gene sequence, genes that are active in the brain appear to exhibit fewer differences between humans and chimpanzees than genes that are active in other tissues. Similarly, genes that are active in many tissues show fewer differences in both sequence and expression level than genes that are active in a single tissue. To explain these findings, the authors propose an additive model whereby each tissue imposes a different level of constraint upon the genes it expresses. These constraints act cumulatively to restrict the evolution of both gene expression and gene sequence in parallel.

It is intuitive that breadth of expression should correlate positively with evolutionary constraint, as genes that are active in many tissues are more likely to participate in cellular processes that are critical for organismal survival and reproduction; indeed, this relationship has been reported previously in a variety of organisms (Duret and Mouchiroud, 2000; Jordan et al, 2004). Less obvious is how the expression of a gene in the brain should impose more evolutionary constraint than expression elsewhere in the body. Here it is important to acknowledge the limitations of the data set and methods. The expression of a gene in prefrontal cortex - but not in heart, liver, kidney, or testis - does not preclude its expression in spleen, lung, muscle, or other tissues; in the absence of a human atlas of gene expression, the notion of tissue-specific constraint is murky. Equally confounding is the contribution of cellular heterogeneity to microarray data interpretation: differences in the number and diversity of cell types between tissues and species may bias the detection of expressed genes (Geschwind, 2000). In a complex tissue such as brain, genes that are widely expressed among neuronal cells are more likely to be detected than low abundance, more cell class limited genes; in this regard, one needs to consider that the apparent lack of diversity and divergence in brain relative to other, more homogeneous tissues may also be explained by a systematic bias based on the genes that are detected. This important issue will be addressed as microarray studies increasingly move to the cellular level (Dougherty and Geschwind, 2005).

Another limitation of the analysis is the focus on protein evolution in terms of single-base-pair substitutions, as comparisons between human and chimpanzee genomic sequence indicate that these changes account for only $\sim 30 \%$ of the observed differences between the species, with the rest coming from insertions, deletions, duplications, and rearrangements (Cheng et al, 2005). These latter types of evolutionary change are usually ignored in comparisons based upon $\mathrm{Ka}$, as genes that do not align well between species are typically not considered. Thus, new frameworks are needed to assess the impact of different types of evolutionary change at a functional level, independent of mechanism. Furthermore, on a gene-by-gene basis, although the correlation between expression and protein divergence is significant, it is very weak $(r<0.05)$, explaining only a small fraction of the variance. This weak correlation is not necessarily surprising, as constraints on evolution at the genome sequence level may not necessarily be reflected in adult gene expression. Rather, sequence differences may correspond more with gene expression at earlier developmental stages, especially in an organ such as the brain, where development has a large impact on function. This is not a fault of the current study, but speaks to the need for additional studies involving different developmental stages.

Comparative evolutionary studies of primates such as the work published by Khaitovich and colleagues are limited primarily by the availability of rare tissue samples. This unfortunate reality can also confound data interpretation. For example, the 30 human tissue samples used in this study were obtained from 30 unique individuals, whereas 
the 25 chimpanzee tissue samples were obtained from only 11 unique individuals, some of whom were related. The only tissues represented by five unrelated individuals, liver and heart, also showed the greatest diversity in gene expression among chimpanzees, raising the possibility that expression diversity attributed to tissues might partially reflect genetic diversity among individuals. It is difficult to dissociate competing effects in this setting, and thus caution should be used when attempting to generalize such findings. Clarification will require replication and the use of additional precious tissue samples. The scarcity of primate tissue is an urgent problem that requires an organized response from the scientific community, as the species from which we stand to learn the most - our closest living relatives, the great apes - are also the most endangered.

We acknowledge grant support from the James S McDonnell Foundation for our collaborative work on human-primate gene expression, the NIMH, and our collaborators Mario Caceres and Todd preuss for their insights and many helpful discussions.

MC Oldham is at the Interdepartmental Program for Neuroscience, and Program in Neurogenetics, Department of Neurology, UCLA School of Medicine, 710 Westwood Plaza, Los Angeles, CA 90095-6814, USA; and DH Geschwind is at Program in Neurogenetics, Department of Neurology and the Neuropsychiatric Institute, UCLA
School of Medicine, 710 Westwood Plaza, Los Angeles, CA 90095-6814, USA

e-mail:dhg@mednet.ucla.edu

Cheng $Z$ et al (2005). Nature 437: 88-93.

Consortium TCSAA (2005). Nature 437: 69-87.

Dougherty D, Geschwind DH (2005). Neuron 45: 183-185 (review).

Duret L, Mouchiroud D (2000). Mol Biol Evol 17: 68-74.

Fay JC, Wu CI (2003). Annu Rev Genomics Hum Genet 4: 213-235.

Geschwind DH (2000). Proc Natl Acad Sci USA 97: 10676-10678.

Jordan IK, Marino-Ramirez L, Wolf YI, Koonin EV (2004). Mol Biol Evol 21: 2058-2070.

Khaitovich P et al (2005). Science 309: 1850-1854.

Khaitovich P et al (2004). PLoS Biol 2: E132.

\section{Further Reading}

Hughes AL (2004). Heredity 93: 5. 